\title{
Parametric Study of a Centrifugal Pump Impeller by Varying the Outlet Blade Angle
}

\author{
E.C. Bacharoudis ${ }^{*}, 1$, A.E. Filios ${ }^{2}$, M.D. Mentzos ${ }^{1}$ and D.P. Margaris ${ }^{1}$ \\ ${ }^{1}$ Fluid Mechanics Laboratory, Mechanical Engineering and Aeronautics Department, University of Patras, Patras, \\ Greece \\ ${ }^{2}$ Fluid Mechanics and Turbomachinery Laboratory, Department of Mechanical Engineering Educators, School of \\ Pedagogical and Technological Education, Athens, Greece
}

\begin{abstract}
The pump design is facilitated by the development of computational fluid dynamics and the complex internal flows in water pump impellers can be well predicted. Various parameters affect the pump performance and energy consumption. The impeller outlet diameter, the blade angle and the blade number are the most critical. The present paper describes the simulation of the flow into the impeller of a laboratory pump in a parametric manner. In this study, the performance of impellers with the same outlet diameter having different outlet blade angles is thoroughly evaluated. The one-dimensional approach along with empirical equations is adopted for the design of each impeller. The predicted performance curves result through the calculation of the internal flow field and a successful correlation of local and global parameters. The numerical solution of the discretized three-dimensional, incompressible Navier-Stokes equations over an unstructured grid is accomplished with a commercial CFD finite-volume code. For each impeller, the flow pattern and the pressure distribution in the blade passages are calculated and finally the head-capacity curves are compared and discussed.
\end{abstract}

Keywords: Centrifugal pump, Impeller, CFD, Numerical study.

\section{INTRODUCTION}

The complexity of the flow in a turbomachine is primarily due to the three dimensional developed structures involving turbulence, secondary flows, unsteadiness etc. Initially, the design of a centrifugal pump was based mainly on empirical correlation, combination of model testing and engineering experience. Nowadays, the design demands a detailed understanding of the internal flow during design and off-design operating conditions. Computational fluid dynamics (CFD) have successfully contributed to the prediction of the flow through pumps and the enhancement of their design.

The complex internal flow at the outlet of the impeller appears a circumferential distortion due to the asymmetric shape of the spiral volute and tongue, especially at off design operating points. Moreover the rotor-volute interaction causes the appearance of dynamic effects which influence the overall pump performance. The non-uniform flow conditions and particularly the pressure field lead to the development of unbalanced radial forces. All these characteristics are crucial for the pump design. The predictions of the performance in combination with the investigation of the complex internal flow through the impeller have developed to fields of intense research.

Various researchers have considerably contributed to revealing the flow mechanisms inside centrifugal impellers with spiral volute or vaned diffuser volute aiming to the

*Address correspondence to this author at the Fluid Mechanics Laboratory, Mechanical Engineering and Aeronautics Department, University of Patras, Patras, Greece; Tel: 0030-2610997193; E-mail: vbach@mech.upatras.gr design of high performance centrifugal turbomachines. The reported works by Eckardt [1], Johnson et al. [2], Kjork et al. [3], Denton [4], Dawes [5], Casey et al. [6], Bansod et al. [7], Krain et al. [8], Farge et al. [9] and Zhang et al. [10] are an indicative collection of research efforts on the computation and the experimental verification of the flowfields within centrifugal impellers.

The last decade research becomes more sophisticated, specifically recently, Hillewaert et al. [11], Gonzalez et al. [12-14], Byskov et al. [15], Meakhail et al. [16], Majidi [17] and Feng et al. [18] extended the prediction of the performance at various operating conditions, different from the normal one, taking into account the dynamic effects of the flow.

Several algorithms have been proposed and developed, targeting to the numerical simulation of the flowfield of a centrifugal impeller. These algorithms apply either pressure based or density based methods for the solution of NavierStokes equations. Lakshminarayana [19], Rodi et al. [20] and Thakur et al. [21], provide a review of the techniques that are useful as an assessment of the state of the art.

It is evident that there is a lot of research work at the numerical and experimental evaluation of the pump flow field, however the study of important manufacturing parameters that influence the performance of a pump is infrequently available in the open literature. Kergourlay et al. [22] studied the influence of adding splitter blades in a hydraulic centrifugal pump impeller. A comparison between impellers with and without splitters and the predicted characteristic curves were presented. Gonzalez et al. [13] tested two centrifugal pump impellers with different outlet diameters for the same volute. A detailed description of the influence of the radial gap between impeller exit and tongue was presented. Anagnostopoulos [23] 
developed a numerical model for the simulation of the 3D turbulent flow and investigated different impeller configurations by varying crucial design parameters.

The present ongoing research is concerned with the influence of the outlet blade angle in the performance of a laboratory centrifugal pump. The design and off-design performance characteristic curves, the local and global variables of the flow field and the resulted non-uniform circumferential pressure field are numerically predicted for three shrouded radial impellers with different outlet blade angle. The computational fluid dynamics analysis is carried out with the commercial software package Fluent ${ }^{\circledast}$ [24], which has been widely used in the field of turbomachinery and the simulation results, have been proven by Sun et al. [25] and Gonzalez et al. [12] to be reliable.

\section{THE PUMP STUDIED}

The systematic research on the influence of the various design aspects of a centrifugal pump in its performance in the whole range of the flow rates requires numerical predictions and experiment. Recently, in the Fluid Mechanics Laboratory of the University of Patras, a pump test rig designed and already is in operation. The test rig is presently equipped with an industrial centrifugal pump that it will be soon replaced by a laboratory pump with the completion of its manufacture. The laboratory pump volute has been especially designed in a way that it can easily suit radial impellers of the same outlet diameter but differ in various main design parameters such as the number of blades, the mean line geometry of the blade, the inlet and outlet blade angles.

The volute of the laboratory centrifugal pump is of rectangular section with rounded corners and its diffuser extends in the radial direction. Three shrouded impellers of constant width $(b=20 \mathrm{~mm})$ with six untwisted blades backward facing have been designed according to Pfleiderer method [26]. The blade length in the three impellers is almost equal. All impellers have the same diameters in suction and pressure side as well as the same blade's leading edge angle $\left(\beta_{1}=14 \mathrm{deg}\right)$ and they vary in the blade's trailing edge angle which is $\beta_{2}=20,30$ and $50 \mathrm{deg}$, respectively. The diameters of the impellers at the suction and pressure side are $D_{1}=150 \mathrm{~mm}$ and $D_{2}=280 \mathrm{~mm}$, respectively.
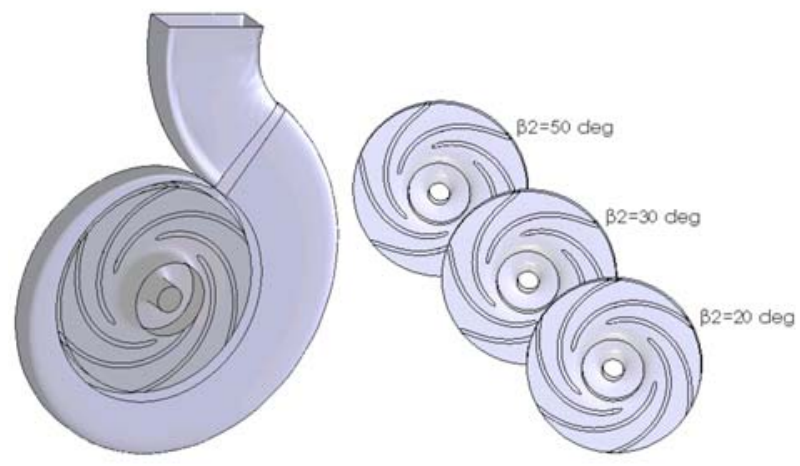

Fig. (1). A 3D image of the laboratory pump with the three radial impellers.
The CAD model of the designed laboratory pump with the three impellers and the absence of their front shroud for blade profile viewing purposes are shown in Fig. (1). In the rotational speed (n) of $925 \mathrm{rpm}$, the normal operation of the three impellers is $0,0125 \mathrm{~m}^{3} / \mathrm{sec}$ flow rate $(\mathrm{Q})$ and according to one dimensional theory the estimated pump's total head $(\mathrm{H})$ is $10 \mathrm{~m}$ that results in the value 18,4 for the specific speed $\left(n_{\mathrm{q}}=\mathrm{n} \cdot \mathrm{Q}^{1 / 2} / \mathrm{H}^{3 / 4}\right)$. At the normal operating point, the hydraulic efficiency $\left(\eta_{H}\right)$ of the pump reaches its maximum value 0,83 .

\section{GOVERNING EQUATIONS}

The incompressible flow through the rotating impeller is solved in a moving frame of reference with constant rotational speed equal the rotational speed of the impeller. The flow through the stationary parts of the pump is solved in an inertial reference frame. The governing equations for the impeller are formulated below

$$
\begin{aligned}
& \nabla \rho \mathbf{u}_{\mathrm{r}}=0 \ldots \\
& \nabla \rho \mathbf{u}_{\mathrm{r}}+2 \rho \boldsymbol{\Omega} \times \mathbf{u}_{\mathrm{r}}+\rho \boldsymbol{\Omega} \times \boldsymbol{\Omega} \times \mathbf{r}=-\nabla \mathrm{p}+\mu_{\text {eff }} \nabla^{2} \mathbf{u}_{\mathrm{r}} \ldots
\end{aligned}
$$

where $\rho$ is the density of the fluid, $p$ is the static pressure, $\mathbf{u}_{\mathrm{r}}$ is the vector fluid velocity in the rotating system, $\boldsymbol{\Omega}$ is the rotational speed and $\mu_{\text {eff }}$ is the dynamic effective viscosity which is a linear combination of laminar and turbulent viscosity derived from $k-\varepsilon$ model of turbulence. The last two terms in the left hand side of equation (2) are the effects of the Coriolis and centrifugal forces due to the rotating frame of reference.

For the stationary parts of the centrifugal pump, the governing equations are formulated in the stationary reference frame. The continuity equation remains the same, but the momentum equation reduces to

$\nabla \rho \mathbf{u}=-\nabla \mathrm{p}+\mu_{\text {eff }} \nabla^{2} \mathbf{u} \ldots$

where $\mathbf{u}$ is the vector fluid velocity in the stationary frame of reference.

The turbulence of the flow is modelled with standard k- $\varepsilon$ model that is rated as the most used model that combines simplicity, robustness and reasonable accuracy. Moreover, it has been tested in a wide range of industrial flows showing satisfactory results. The differential transport equations for the turbulence kinetic energy and turbulence dissipation rate are:

$$
\begin{aligned}
& \nabla \rho \mathbf{u k}=\nabla\left(\left(\mu+\frac{\mu_{\mathrm{t}}}{\sigma_{\mathrm{k}}}\right) \nabla \mathrm{k}\right)+\mathrm{G}_{\mathrm{k}}-\rho \varepsilon \ldots \\
& \nabla \rho \mathbf{u} \varepsilon=\nabla\left(\left(\mu+\frac{\mu_{\mathrm{t}}}{\sigma_{\varepsilon}}\right) \nabla \varepsilon\right)+\mathrm{C}_{1 \varepsilon} \frac{\varepsilon}{\mathrm{k}} \mathrm{G}_{\mathrm{k}}-\mathrm{C}_{2 \varepsilon} \rho \frac{\varepsilon^{2}}{\mathrm{k}} \ldots \\
& \mu_{\mathrm{t}}=\rho \mathrm{C}_{\mathrm{m}} \frac{\mathrm{k}^{2}}{\varepsilon} \ldots
\end{aligned}
$$

where $\mathbf{u}$ is the local velocity vector, $\mathrm{k}$ is the turbulent kinetic energy, $\varepsilon$ is the dissipation rate, $\mu$ is the laminar viscosity, $\mu_{t}$ is the turbulent viscosity, $G_{k}$ represents the generation of turbulent kinetic energy due to the mean velocity gradients, $\sigma_{\kappa}$ 
and $\sigma_{\varepsilon}$ are the turbulent Prandtl numbers and $\mathrm{C}_{1 \varepsilon}=1,44$, $\mathrm{C}_{2 \varepsilon}=1,92$ and $\mathrm{C}_{\mathrm{m}}=0,09$ are the constants of the model.

\section{COMPUTATIONAL ISSUES}

\section{Geometry and Grid}

The numerical treatment of the radial pump implies the spatial discretization of the flow domain. All gaps between the impeller shroud and the pump casing are neglected in the present numerical simulation. The whole domain consists of three sub-domains or zones. The first and third zones are stationary while the second zone that incorporates the blade is moving with the applied rotational speed of $\mathrm{n}=925 \mathrm{rpm}$.

The first zone represents the suction or inlet pipe that is $100 \mathrm{~mm}$ in diameter and the third zone is the discharge or outlet portion where the flow is fully developed with a less possible reacting outlet boundary condition. The intermediate zone consists of the eye and the impeller of the pump. The three sub-domains were separated further by additional inner faces forming different blocks. In this way, the density and the quality of the cells in local flow field regions can be suitably controlled and handled depending on pressure gradients and velocities.

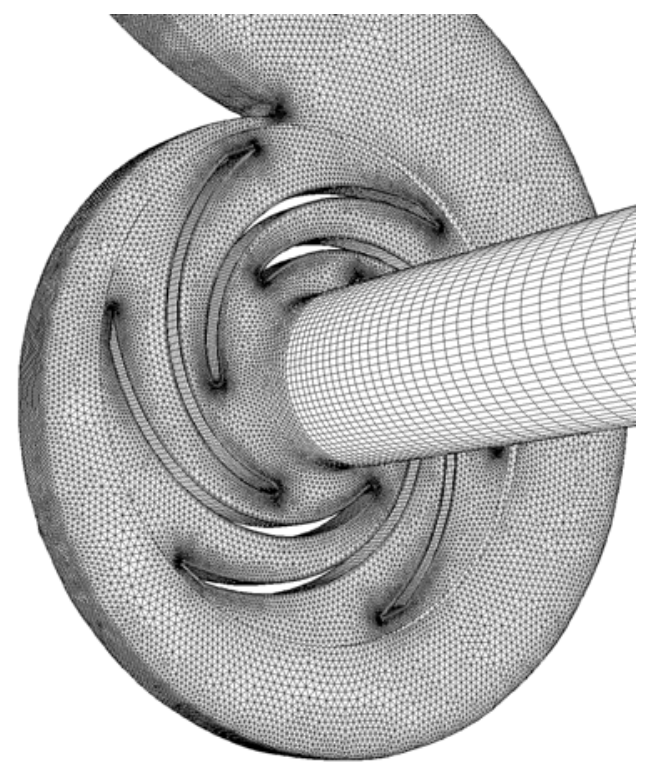

Fig. (2). Sketch of the unstructured mesh of the pump.

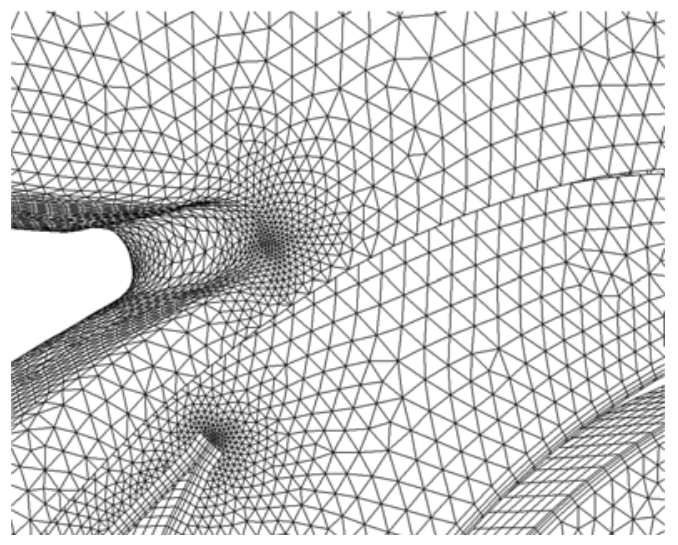

Fig. (3). Details of the unstructured mesh in the region of the tongue.

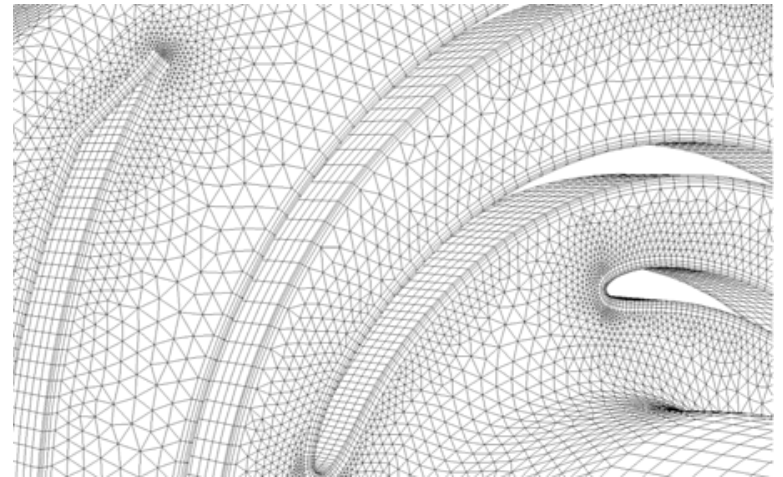

Fig. (4). The structured hexahedral cells around the blades combined with the unstructured mesh in the blade passage.

The geometry and the mesh of the computational pump domain were generated with Fluent's pre-processor, Gambit ${ }^{\boxplus}$ [27]. Unstructured wedges are generated to define the inlet and outlet zones. An unstructured mesh with tetrahedral cells is also used for the zones of impeller and volute as shown in Fig. (2). The mesh is refined in the near tongue region of the volute (see Fig. (3)), as well as in the regions close to the leading and trailing edge of the blades. Around the blades, structured hexahedral cells are generated, as shown in Fig. (4). Though the size of the cells in the wall regions is not adequate to resolve the viscosity-affected region inside the boundary layer, the appropriate number of cells exists inside the boundary layer for the approach of standard wall functions. The latter provides correct values for the pump performance and allows a detailed analysis of the main phenomena involved.

\section{Remarks on the Numerical Simulation}

All the calculations have been performed with Fluent ${ }^{\circledR}$ CFD software package [24] that utilizes the finite volume method for the solution of the steady 3D incompressible Navier-Stokes equations, including the centrifugal force source in the impeller. Turbulence is modelled with the selection of the standard $\mathrm{k}-\varepsilon$ model. The involved parameters regarding the turbulence intensity and the hydraulic diameter, in the lack of realistic turbulent inflow conditions in industrial applications, are estimated with values of $5 \%$ and $\mathrm{D} / 2$, respectively. The pressure-velocity coupling is performed through the SIMPLE algorithm. Second order, upwind discretization is used for convections terms and central difference schemes for diffusion terms.

The applied boundary conditions involve the extension of the computational domain by adding a reasonable length at the inlet and outlet pipes of the centrifugal pump in order to better simulate the pumping circuit influence. At the inlet zone, the axial velocity is a constant based on the through flow for the pump. The absolute tangential velocity at the inlet is zero, which implies, in the rotating frame, that the relative velocity is $-\mathrm{r} \boldsymbol{\Omega}$ and the radial velocity is zero. At the exit of the discharge pipe, assuming a fully developed turbulent flow, a practically zero velocity gradient is set. The walls of the model are stationary with respect to their respective frame of reference, and the no slip condition is applied.

Although grid size is not adequate to investigate local boundary layer variables, global ones are well captured. For such calculations wall functions, based on the logarithmic law, have been used. 
Since the problem involves both stationary and moving zones, the multiple reference frame model has been selected. It is a quasi-steady state approximation in which individual cell zones move at different rotational speeds. As the rotation of the reference frame and the rotation defined via boundary conditions can lead to large complex forces in the flow, calculations may be less stable as the speed of rotation and hence the magnitude of these forces increases. To control this undesirable effect, each run starts with a low rotational speed and then the rotation is slowly increased up to the desired level.

The simulations were executed in a $3 \mathrm{GHz}$ Pentium IV PC. The number of iterations adjusted to reduce the scaled residual below the value of $10^{-5}$, which is the criterion of convergence. For each run, the observation of the integrated quantities of total pressure, at suction as well as at discharge surface was appointed for the convergence of the solution. In many cases this drives the residuals in lower values than the initially set value. Depending on the case, the convergence was achieved at different iterations, as the result at a specific mass-flow was used to initialize the computations at another mass-flow. Aiming to smooth convergence, various runs were attempted by varying the under-relaxations factors. In that way a direct control regarding the update of computed variables through iterations, was achieved. Initializing with low values for the first iterations steps and observing the progress of the residuals, their values were modified for accelerating the convergence.

\section{NUMERICAL RESULTS}

The outlet angle of three different impellers is correlated with the slope of the H-Q performance curve of each impeller. Increasing the outlet blade angle, the shape of the curve becomes smoother and flatter. This fact is expected and is consistent with theory, i.e.

$\mathrm{H}=\mu \eta_{\mathrm{H}} \frac{\mathrm{u}_{2}^{2}}{\mathrm{~g}}\left(1-\frac{\mathrm{c}_{\mathrm{m} 3}}{\mathrm{u}_{2}} \cot \beta_{2}\right) \ldots$

where $\mu$ is the slip factor [28], $\eta_{H}$ is the hydraulic efficiency, $\mathrm{u}_{2}$ is the peripheral velocity at the outlet section of the impeller, $\mathrm{c}_{\mathrm{m} 3}$ is the meridian velocity at the exit of impeller passage and $\beta_{2}$ is the outlet blade angle.

Equation (7) enables the ascertainment of the effect of the two design parameters $\mathrm{c}_{\mathrm{m} 3} / \mathrm{u}_{2}$ and $\beta_{2}$ on the total head. If $\mathrm{c}_{\mathrm{m} 3} / \mathrm{u}_{2}$ is reduced, then the total head is increased. Certainly, the effect of the vane discharge angle is not so straightforward. If the $\beta_{2}$ angle increases, then the total head increases, too. However, the effect of increase the angle $\beta_{2}$ is partly cancelled since the slip factor formulas indicate that larger angle $\beta_{2}$ results to the decrease of the slip factor value. The variation of the slip factor affects the shape of the H-Q curve and it is noticeable for blade angles grater than $25 \mathrm{deg}$.

The later is in agreement with the results of the numerical prediction of the H-Q curves for the examined impellers, which are shown in Fig. (5a). The ordinate is the non-dimensional total head of the pump while the abscissa is the non-dimensional flow rate. The $\mathrm{Q}_{\mathrm{N}}=58,5 \mathrm{~m}^{3} / \mathrm{h}$ and
$\mathrm{H}_{\mathrm{N}}=8,93 \mathrm{~m}$ are the CFD predicted nominal volume rate and the nominal total head for the $\beta_{2}=20 \mathrm{deg}$ impeller, respectively.

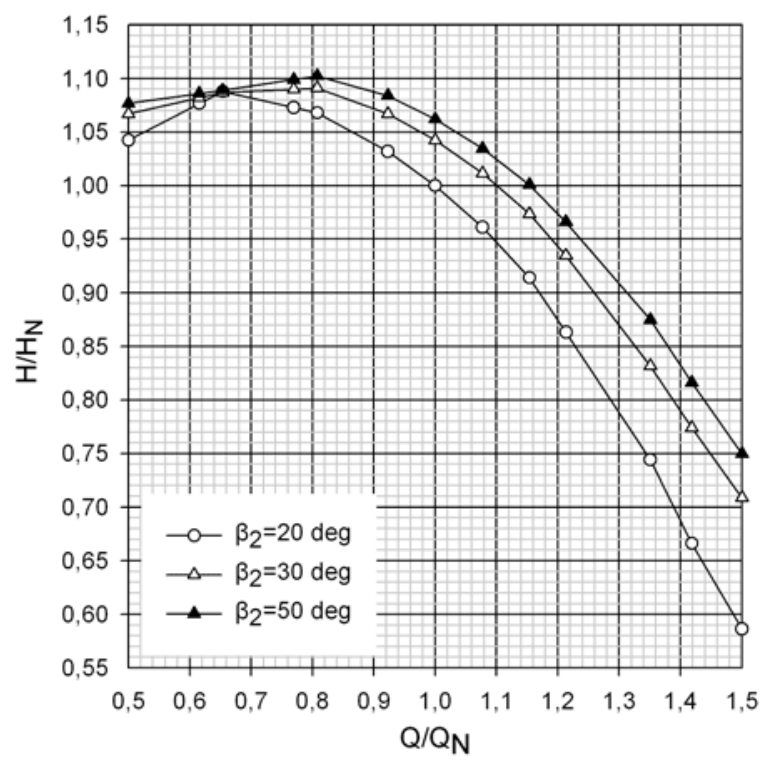

Fig. (5a). Predicted head curves for the examined pump impellers.

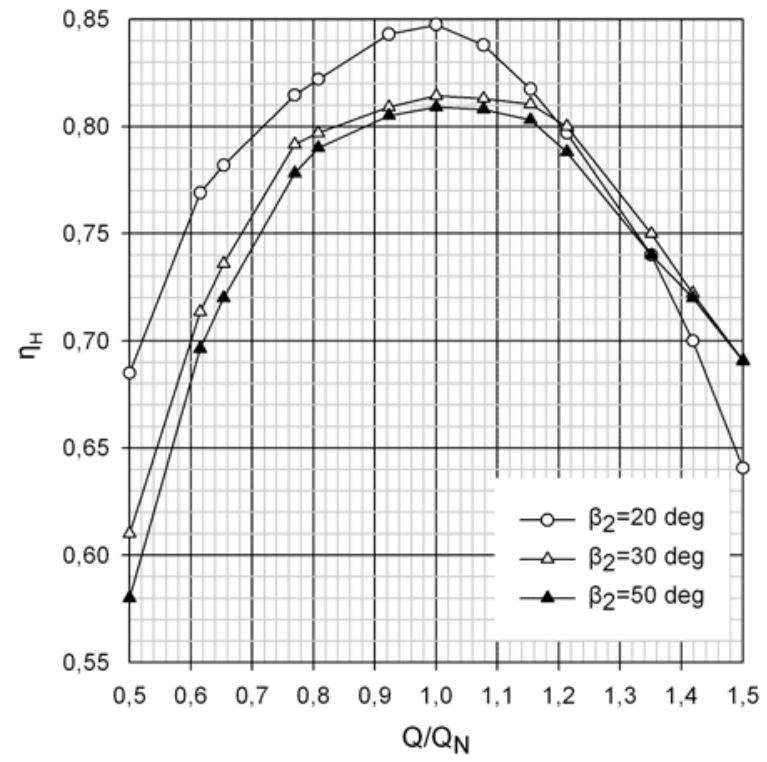

Fig. (5b). Predicted hydraulic efficiency curves for the examined pump impellers.

The nominal flow rate is defined by the point in which the hydraulic efficiency of the pump reaches its maximum value. The variation of the hydraulic efficiency against the non dimensional flow rate for the numerically studied impellers, are shown in Fig. (5b). At the nominal flow rate the value of the hydraulic efficiency is ranged between 0,81 and 0,845 for the three impellers which is in reasonable accuracy with the predicted value according to the applied design method. A $50 \%$ reduction of the volume rate from its nominal value results to $20 \%, 25 \%$ and $28 \%$ drop of the $\eta_{\mathrm{H}}$ relative to their nominal value for the impellers with $\beta_{2}=20,30$ and 50deg respectively. A $50 \%$ increase of the volume rate from the nominal point leads to $25 \%, 15 \%$ and $15 \%$ drop of the $\eta_{H}$ relative to the nominal one for the three impellers respectively. 
The curve of the hydraulic efficiency for $\mathrm{Q}<\mathrm{Q}_{\mathrm{N}}$ decreases more rapidly for the impellers with $\beta_{2}=30 \mathrm{deg}$ and $50 \mathrm{deg}$ than for $\beta_{2}=20 \mathrm{deg}$. The opposite happens for $\mathrm{Q}>\mathrm{Q}_{\mathrm{N}}$ where the $\eta_{\mathrm{H}}$ curve of the $\beta_{2}=20 \mathrm{deg}$ impeller is steeper. The comparison of the hydraulic efficiency of the three impellers at $\mathrm{Q}=\mathrm{Q}_{\mathrm{N}}$ shows that the increase of the outlet angle more than $10 \mathrm{deg}$ till to $30 \mathrm{deg}$ reduce it almost $3 \%$.

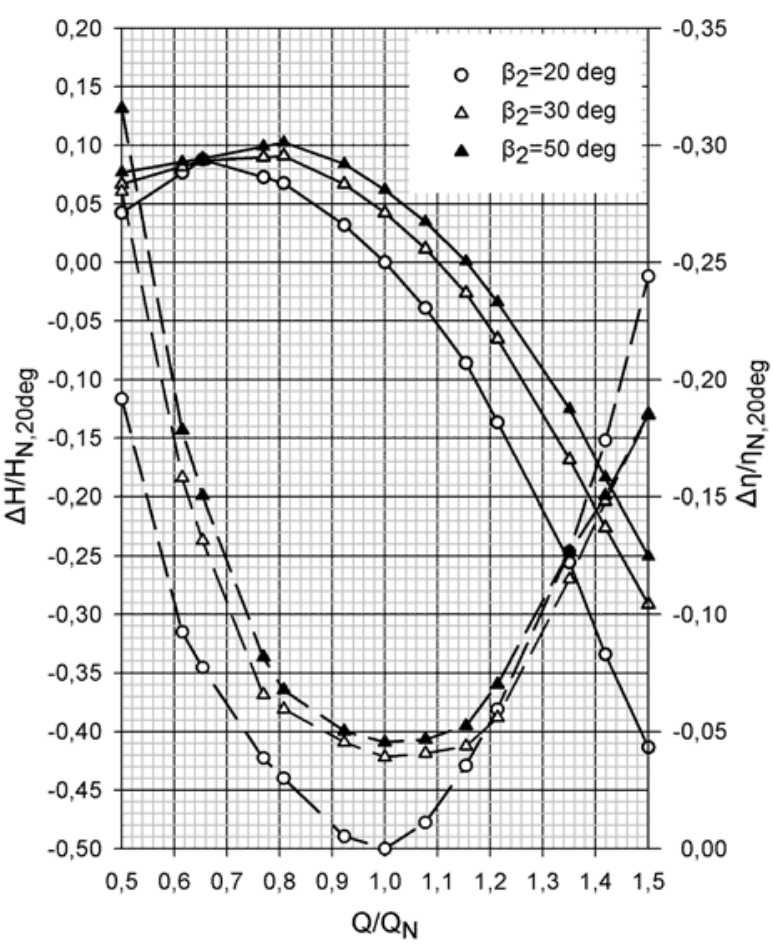

Fig. (6). Percentage variation of the head (solid lines) and hydraulic efficiency (dash lines) curves with outlet blade angle for the examined impellers.

The shift of the performance curves due to the variation of the outlet blade angle may be expressed in terms of percentage variation with reference of the corresponding values $\mathrm{H} / \mathrm{H}_{\mathrm{N}}$ and $\eta_{\mathrm{H}}$ at nominal capacity for the $\beta_{2}=20 \mathrm{deg}$ impeller, as shown in Fig. (6). Thus, at nominal capacity, a $10 \mathrm{deg}$ increase of the $\beta_{2}$ angle causes a $4,2 \%$ increase of the head and a 3,9\% decrease of the hydraulic efficiency. If the $\beta_{2}$ increases $30 \mathrm{deg}$, i.e. from $20 \mathrm{deg}$ to $50 \mathrm{deg}$, then the head increases $6,2 \%$ and the hydraulic efficiency decreases $4,5 \%$.

Besides the predicted performance curves of the studied impellers, the assessment of the local characteristics of the internal flow field is accomplished. The static pressure fields for the plane near the back shroud for the two of the studied impellers are shown in Fig. (7). At constant radial position, it is confirmed that the static pressure drops from the pressure side to the suction side of the impeller blade and this pressure drop reduces at the exit of the blade passage. Moreover, the static pressure patterns are not the same in the planes between the hub and the shroud. The variation is noticed in the pressure contour field of the lateral surfaces of the blades. The observed pressure variation does not entail any additional losses in the pump and simply implies that each blade can only transmit a fixed amount of energy and certainly it is lower than the value prescribed by Euler's equation. Qualitatively, the patterns are similar for the three examined impellers. As it is expected, the variations are focused on the static pressure values that become larger with the increase of the outlet blade angle.

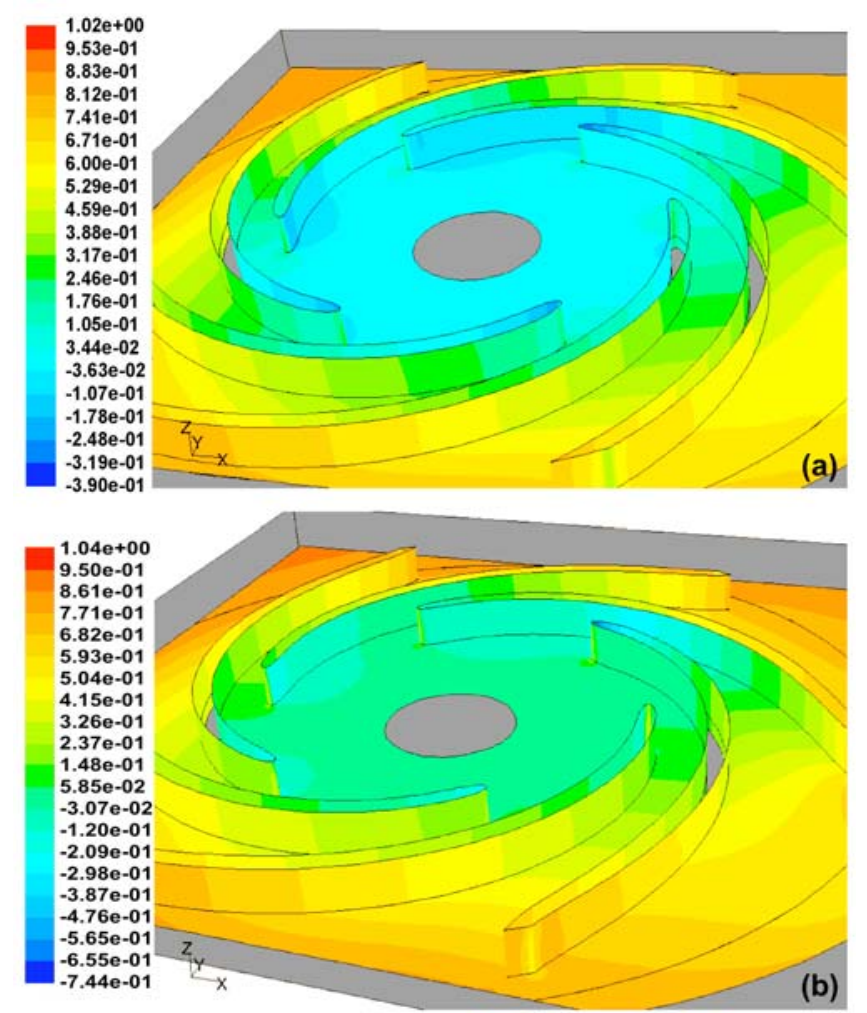

Fig. (7). Relative static pressure contours (atm) at $Q_{N}$ for the $\beta_{2}=20$ $\operatorname{deg}(\mathrm{a})$ and $\beta_{2}=50 \mathrm{deg}(\mathrm{b})$ impellers.

The minimum value of the static pressure inside the impeller is located at the leading edge of the blades at the suction side except for the blade with trailing edge, which end up at the region of the tongue. For that blade, the minimum pressure is located at the leading edge of the blade at the pressure side primarily due to the blade - tongue interaction. Another reason for the appearance of low local pressures at the pressure side of the blade is related with the geometry and the design method of the blade, which permits the development of such pressure contours. Hence, the minimum pressure is observed at these regions. The same situation stands for flow rates greater or lower than the nominal one, as well as for different outlet angles.

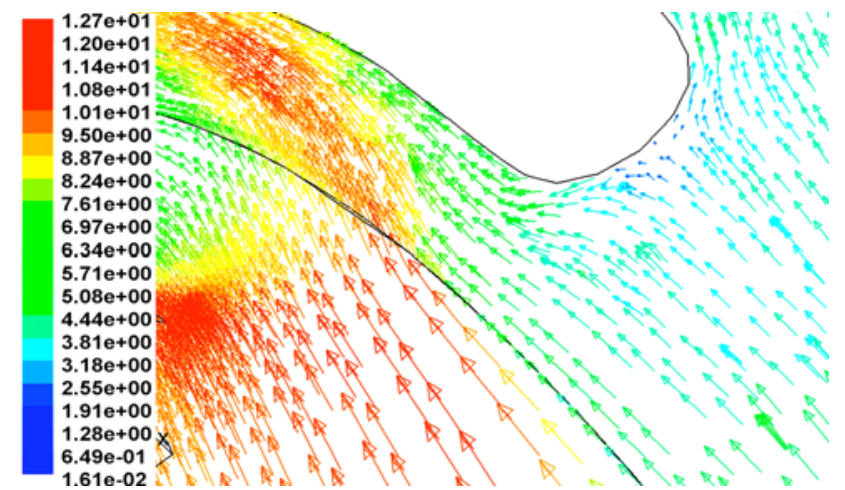

Fig. (8). The absolute velocities $(\mathrm{m} / \mathrm{s})$ in the region of the tongue for the $\beta_{2}=20$ deg impeller. 


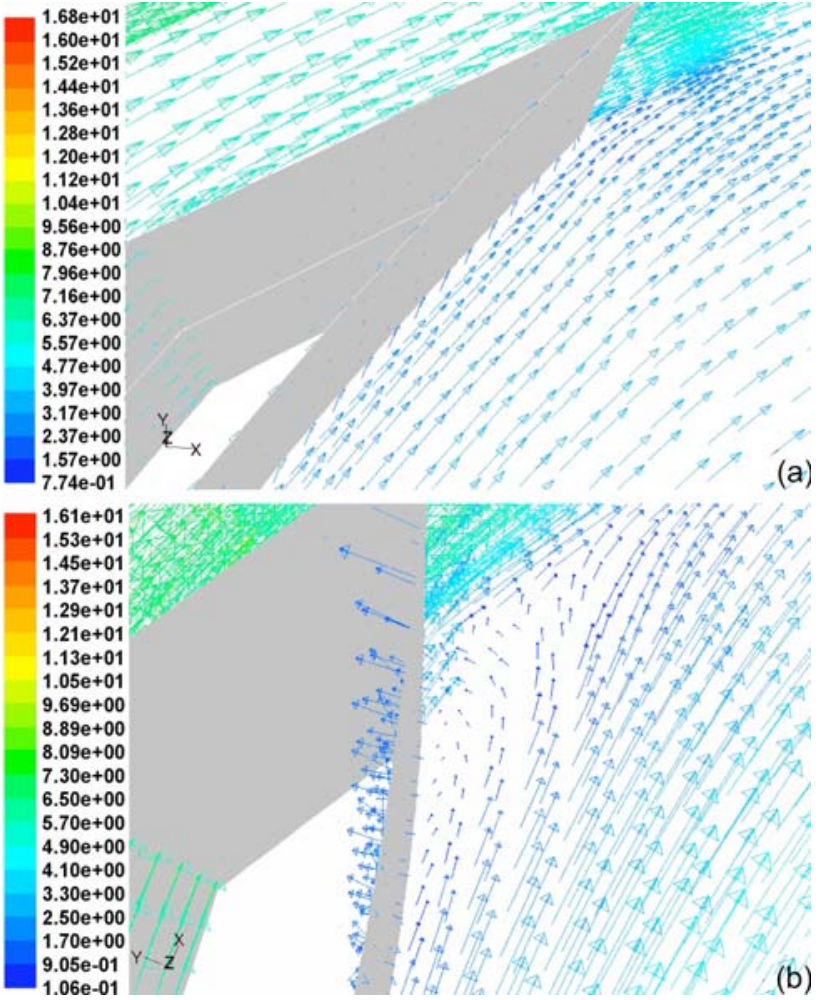

Fig. (9). The relative velocities $(\mathrm{m} / \mathrm{s})$ at the trailing edge of the blade with $\beta_{2}=20 \mathrm{deg}$ (a) and $\beta_{2}=50 \mathrm{deg}$ (b).

The flow close to the tongue and at the design operation is shown in Fig. (8). The pattern verifies that the position of the stagnation point is placed in the middle of the tongue edge and it is in agreement with published data [13]. Furthermore, the outlet blade angle affects and changes the relative velocity patterns. As the $\beta_{2}$ increases, a recirculation zone establishes at the trailing edge of the blade as Fig. (9) shows.

Significant variations in the absolute velocity field in the blade passage are displayed when the impeller operates offdesign. Fig. (10) shows the absolute velocity vectors in the leading edge of one blade for the three studied impellers with each one operating in nominal capacity or at the edges of the examined capacity range. When the volume flow rate is nominal or more than nominal, the fluid flows smoothly through the impeller passage, except for the flow passage which ends up at the region of tongue. The flow direction in combination with the blade curvature exhibits a weak vortex at the pressure side of the blade just downstream the leading edge giving reasons to the appearance of low pressure in this region. Reversely, when the impeller operates in volume flow rates less than nominal, a recirculation zone is established in the leading edge of each blade.

The absolute velocity patterns which are shown in Fig. (10) do not suggest that the velocity distribution is uniform in the blade passage across the impeller width. Non uniformities are mainly present in the region of the leading edge and these are minimized in the outlet of the impeller. The unevenness velocity distribution in the entrance of the blade passage is due to the different wall shape of the hub and shroud as well as to inability of the fluid to adjust its path in the imposed entrance geometry. The fluid elements in the inside of the turn have

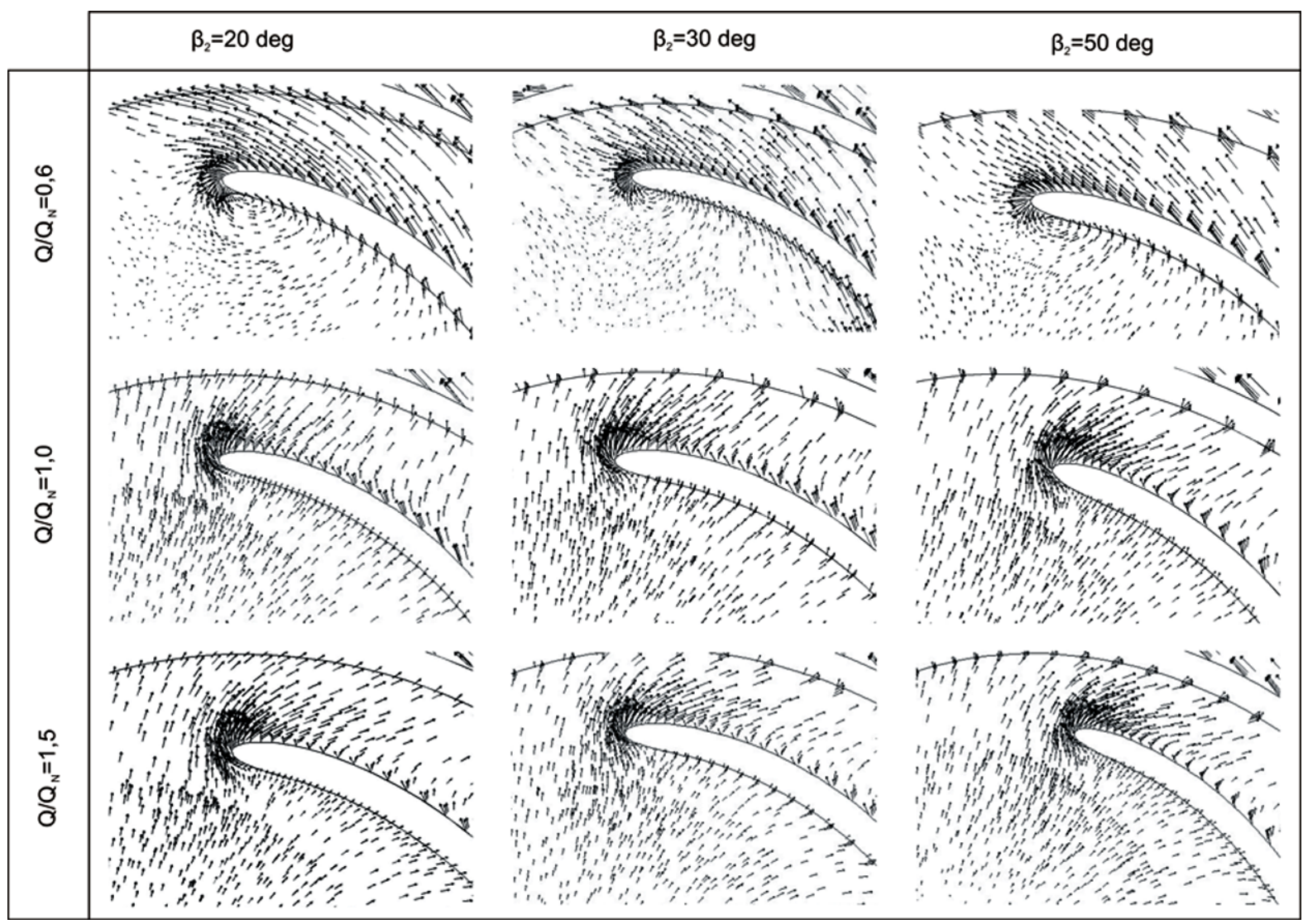

Fig. (10). Captures of the absolute velocity patterns in the region of leading edge at the middle span plane for the three studied impellers in design and off-design operation. 
smaller velocities than those in the outside of curvature. These non uniformities in the velocity distribution are amplified when the pump operates off-design and especially when $\mathrm{Q}$ is less than $\mathrm{Q}_{\mathrm{N}}$ where moreover the aforementioned inlet recirculation establishes.
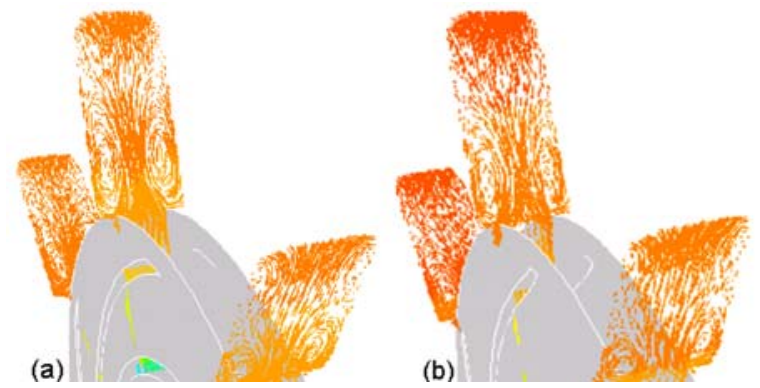

Fig. (11). Secondary flow patterns at nominal flow in three planes along the volute that suits the $\beta_{2}=20 \mathrm{deg}$ (a) and $\beta_{2}=50 \mathrm{deg}$ (b) impellers.

Published research work [14] supports the presence of secondary flow along the duct of the volute, which detected for all impellers and all volume flow rates. Fig. (11) shows a capture of the flow in the volute of the pump that operates in nominal flow rate and equipped with two of the studied impellers. As the fluid approaches the discharge, eddies are maintained with small changes of their size and core position.

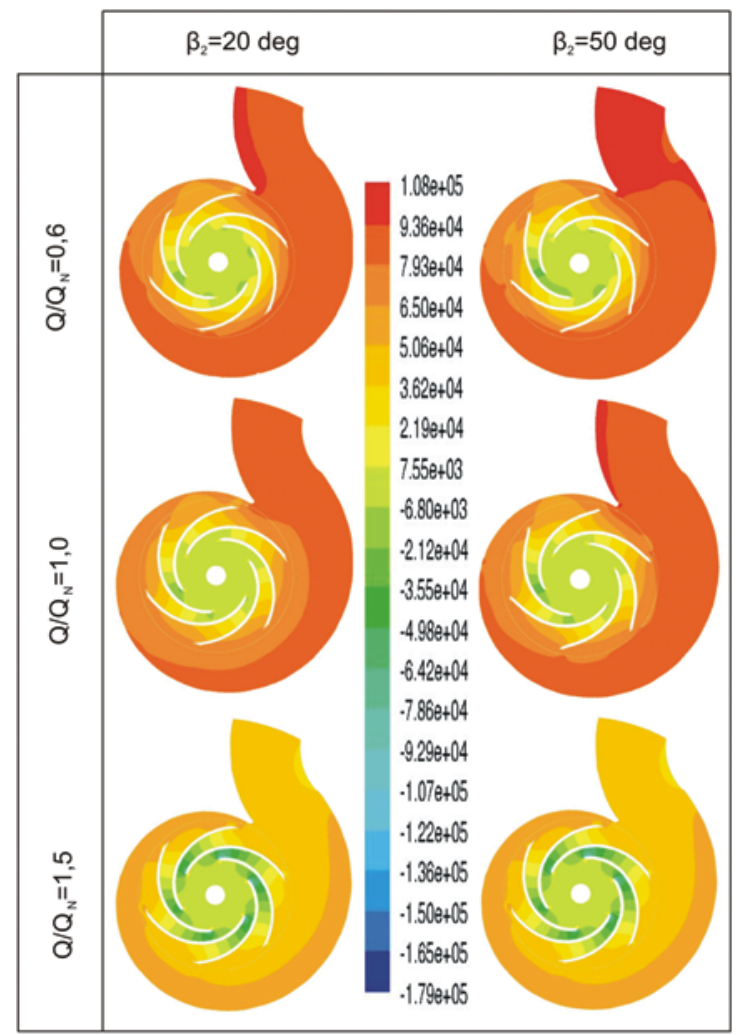

Fig. (12). The relative static pressure (atm) contours inside the laboratory pump.

The static pressure variation within the pump, for three flow rates and for two different outlet blade angles, is shown in Fig. (12). The pressure forces seem to be the main driven mechanism to establish the flow features both in the impeller and spiral volute. Fig. (13) shows the computed pressure distributions around the impeller periphery for the three examined impellers in four volume flow rates. Both figures confirm the non uniform pressure contours at the outlet of the impellers which are consistent with the global characteristics of the impellers and their performance.
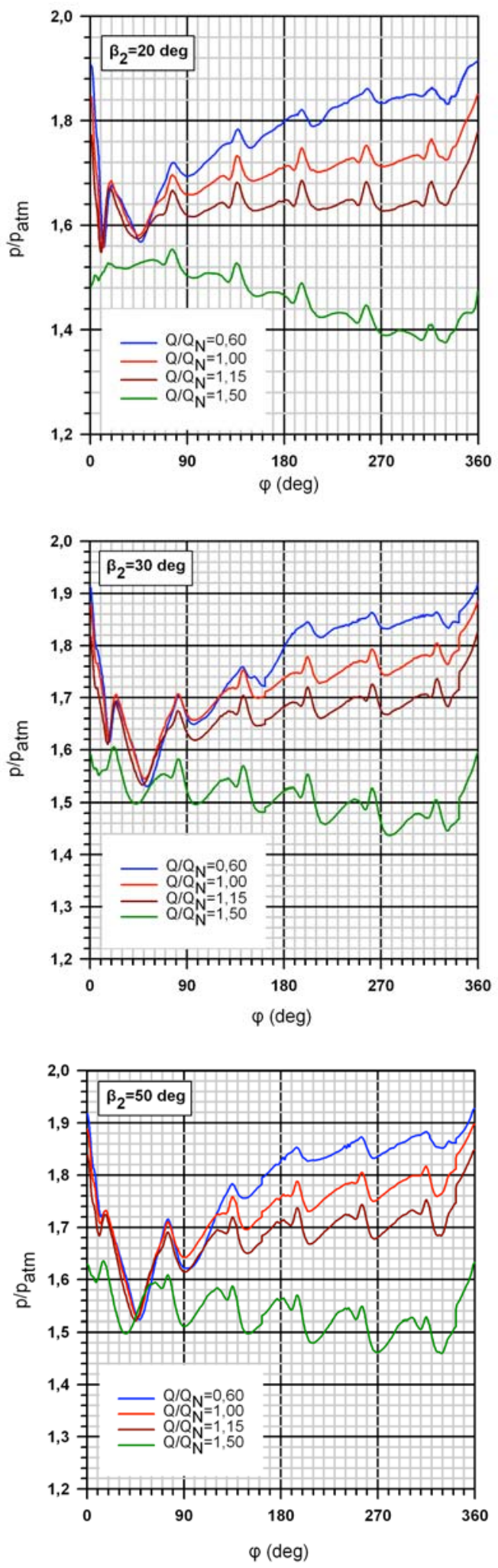

Fig. (13). Pressure distributions at the periphery of the studied impellers in four different flow rates. 
For flow rates lower than nominal, the pressure reaches its lowest value directly in front of the tongue and starts to increase around the periphery of the impeller, taking its maximum value just behind the tongue. When the flow rate approaches the nominal, the pressure distribution is smoothed achieving the most uniform contour in nominal volume flow rate. At higher flow rates, the pressure decreases gradually from its maximum value in front of the tongue to a minimum value just behind the tongue. The aforementioned verify remarkable influence of the tongue in the pressure field inside the pump.

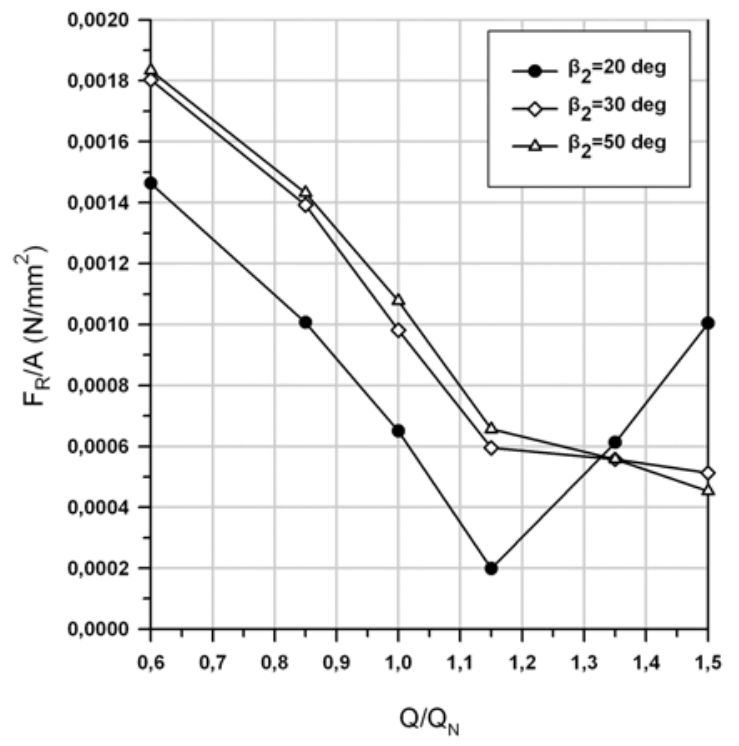

Fig. (14). Computed radial forces in the examined impellers in design and off-design operation.

The non uniform static pressure field around the impeller results in a radial force which is calculated by the integration of the pressure force distribution. For the studied impellers operating in nominal volume flow rate and away from it, the computed radial forces $\left(\mathrm{F}_{\mathrm{R}}\right)$ divided by the impeller shroud area (A) are shown in Fig. (14).

For the $\beta_{2}=20 \mathrm{deg}$ impeller with its standard volute casing, a "V" shape diagram of the radial force is obtained. For volume flow rates close to the nominal, a more uniform pressure distribution exists which implies less radial forces. On the other hand, far from the design operation the non uniformity is quite profound which results in larger forces. The minimum radial forces for that case were calculated near the best efficiency point as expected. The same trend is observed for the other two impellers with outlet blade angle $\beta_{2}=30$ and $50 \mathrm{deg}$. It is remarkable the shift of the minimum radial force to higher flow rates. The shift from the nominal point has been reported by researchers in literature and it has been confirmed with experiments [13]. It is explained by the fact that the pressure distribution Fig. (13) becomes more uniform as the exit angle of the blade increases in high flow rates. However, the simplifications of the computational pump geometry in comparison with the real one (no sided rooms for leakage flow) and the steady state approach should be taken into consideration because they affect the position of the minimum force. When $\mathrm{Q} \approx 1,5 \mathrm{Q}_{\mathrm{N}}$ the pressures around the $\beta_{2}=30$ and $50 \mathrm{deg}$ impellers are varied almost around a mean value, which is not observed for $\beta_{2}=20 \mathrm{deg}$ impeller. For blade angles above $30 \mathrm{deg}$ and flow rates higher than 1,5 the radial force increases, displaying the characteristic "V" shape.

\section{CONCLUDING REMARKS}

A laboratory pump that can suit radial impellers with the same diameter has been designed. Initially, three shrouded impellers with outlet blade angle $20 \mathrm{deg}, 30 \mathrm{deg}$ and $50 \mathrm{deg}$ respectively were designed and with the aid of computational flow dynamics, the flow patterns through the pump as well as its performance for flow rates in design and off-design operation are predicted. On the assumption of the one dimensional flow theory, the pump was designed to operate at nominal characteristics $\mathrm{Q}=45 \mathrm{~m}^{3} / \mathrm{h}$ and $\mathrm{H}=10 \mathrm{~m}$, when the rotational speed is $925 \mathrm{rpm}$.

The CFD predicted value of the head at the nominal flow rate is approximately $\mathrm{H}=9 \mathrm{~m}$. There is a shift of the numerical nominal flow rate towards greater values and a discrepancy of $10 \%$ between the theoretical head and the predicted numerical head. One reason for this shift is due to the fact that Pfleiderer design method does not take care of the complicated three dimensional flow structures and empirical formulas are applied for the account of hydraulic losses. Consequently, the increase of the nominal flow rate causes a reduction in the total head of the pump. So the discrepancy between theoretical and numerical values can be explained.

The numerical simulations seem to predict reasonably the total performance and the global characteristics of the laboratory pump. The influence of the outlet blade angle on the performance is verified with the CFD simulation. As the outlet blade angle increases the performance curve becomes smoother and flatter for the whole range of the flow rates. When pump operates at nominal capacity, the gain in the head is more than $6 \%$ when the outlet blade angle increases from 20 deg to $50 \mathrm{deg}$. However, the above increment of the head is recompensed with $4,5 \%$ decrease of the hydraulic efficiency. When the pump operates off-design, the percentage raise of the head curve, due to the increment of the outlet blade angle, is larger for high flow rates and becomes smaller for flow rates $\mathrm{Q} / \mathrm{Q}_{\mathrm{N}}<0,65$. Moreover, at high flow rates, the increase of the outlet blade angle causes a significant improvement of the hydraulic efficiency.

Further research work is planned to complete this study through the validation of the CFD predictions with experimental data and simultaneously to investigate other crucial design parameters for the laboratory pump performance.

\section{REFERENCES}

[1] D. Eckardt, "Detailed flow investigations within a high-speed centrifugal compressor impeller", ASME Journal of Fluids Engineering, vol. 98, pp. 390-402, 1976.

[2] M. W Johnson, and J. Moore, "The development of wake flow in a centrifugal impeller", ASME Journal of Engineering for Power, vol. 102, pp. 382-390, 1980.

[3] A. Kjork, and L. Lofdahl, "Hot-wire measurement inside a centrifugal impeller", ASME Journal of Fluids Engineering, vol. 111, pp. 363368, 1989.

[4] J.D. Denton, "The calculation of three-dimensional viscous flow through multistage turbomachinery", ASME Journal of Turbomachinery, vol. 114, pp. 18-26, 1992. 
[5] M.W. Dawes, "Toward improved through flow capability: the use of three-dimensional viscous flow solvers in a multistage environment", ASME Journal of Turbomachinery 114, pp. 8-17, 1992.

[6] M.V. Casey, P. Dalbert, and P. Roth, "The use of 3D viscous flow calculations in the design and analysis of centrifugal compressors", ASME Journal of Turbomachinery, vol. 114, pp. 27-37, 1992.

[7] P. Bansod, and C.M. Rhie, "Computation of flow through a centrifugal impeller with tip leakage", AIAA Paper No 90-2021, 1990.

[8] H. Krain, and W. Hoffman, "Verification of an impeller by laser measurement and 3D viscous flow calculations", ASME Paper 89-GT-150, 1989.

[9] T.Z. Farge, and M.W. Johnson, "The effect of backswept blading on the flow in a centrifugal compressor impeller", ASME Paper 90-GT-231, 1990.

[10] M.J. Zhang, C.G. Gu, and Y.M. Miao, "Numerical study of the internal flow field of a centrifugal impeller", ASME Paper 94GT-357, 1994.

[11] K. Hillewaert and R.A. Van den Braembussche, "Numerical simulation of impeller-volute interaction in centrifugal compressors", ASME Journal of Turbomachinery, vol. 121, pp. 603-609, 1999.

[12] J. Gonzalez, J. Fernandez, E. Blanco, and C. Santolaria, "Numerical simulation of the dynamic effects due to impellervolute interaction in a centrifugal pump", ASME Journal of Fluids Engineering, vol. 124, pp. 348-355, 2002.

[13] J. Gonzalez, J. Parrondo, C. Santolaria, and E. Blanco, "Steady and unsteady forces for a centrifugal pump with impeller to tongue pump variation", ASME Journal of Fluids Engineering, vol. 128, pp. 454-462, 2006.

[14] J. Gonzalez, and C. Santolaria, "Unsteady flow structure and global variables in a centrifugal pump", ASME Journal of Fluids Engineering, vol. 128, pp. 937-946, 2006.

[15] R. K. Byskov, C. B. Jacobsen, and N. Pedersen, "Flow in a centrifugal pump impeller at design and off-design conditionsPart II: Large Eddy Simulations", ASME Journal of Fluids Engineering, vol. 125, pp. 73-83, 2003.
[16] T. Meakhail and S.O. Park, "A study of impeller-diffuser-volute interaction in a centrifugal fan", ASME Journal of Turbomachinery, vol. 127, pp. 84-90, 2005.

[17] K. Majidi, "Numerical study of unsteady flow in a centrifugal pump", ASME Journal of Turbomachinery, vol. 127, pp. 363-371, 2005.

[18] J. Feng, F.K. Benra and H.J. Dohmen, "Numerical investigation on pressure fluctuations for different configurations of vaned diffuser pumps", International Journal of Rotating Machinery, vol. 2007, 2007.

[19] B. Lakshminarayana, "An assessment of computational fluid dynamic techniques in the analysis and design of turbomachinery", ASME Journal of Fluids Engineering, vol. 113, pp. 315-352, 1991.

[20] W. Rodi, S. Majumdar, and B. Schonung, "Finite volume methods for two-dimensional incompressible flows with complex boundaries", Computer Methods in Applied Mechanics and Engineering, vol. 75, pp. 369-392, 1989.

[21] S. Thakur, J. Wright, W. Shyy, J. Liu, H. Ouyang, and T. Vu, "Development of pressure-based composite multigrid methods for complex fluid flows", Program in Aerospace Science, vol. 32, pp. 313-375, 1996.

[22] G. Kergourlay, M. Younsi, F. Bakir and R. Rey, "Influence of splitter blades on the flow field of a centrifugal pump: Test-analysis comparison", International Journal of Rotating Machinery, vol. 2007, 2007.

[23] J. Anagnostopoulos, "CFD Analysis and design effects in a radial pump impeller", WSEAS Transactions on Fluid Mechanics, vol. 1 (7), pp. 763-770, 2006.

[24] Fluent Inc., http://www.fluent.com/.

[25] J. Sun, and H. Tsukamoto, "Off-design performance prediction for diffuser pumps", Journal of Power and Energy, Proceedings of I. Mech. E., Part A, vol. 215, pp. 191-201, 2001.

[26] C. Pfleiderer, Die Kreiselpumpen für Flüssigkeiten und Gase. Springer-Verlag, Berlin, 1961.

[27] Gambit: Computational Fluid Dynamics (CFD) Preprocessor, http://www.fluent.com/software/gambit

[28] D. Japikse, W.D. Marscher and R.B. Furst, Centrifugal Pump Design and Performance. Concepts ETI, Inc., Vermont, USA, 1997. 